\title{
Putting evolution to good use
}

\author{
Even materials scientists have reason to celebrate the seminal insights of Charles Darwin on \\ his bicentenary.
}

This year, Charles Darwin is everywhere. The man who solved the puzzle of evolution was born 200 years ago, and it is 150 years since the publication of On the Origin of Species. Darwin's work transformed our understanding of the natural world and our place within it, but one might be forgiven for thinking that this scarcely has much relevance to the science and engineering of materials.

Yet Darwin's explanation for how complex form evolved in the living world is very much a matter of engineering. As biologist Steve Jones puts it in his new book Darwin's Island (Little, Brown, London, 2009), 'Natural selection is a factory that makes almost impossible things. It manufactures what seems like design with no need for a designer.' Put that way, the process of evolution no longer seems a purely biological phenomenon, but begins to sound potentially useful to the engineer.

It requires two things: random variation, which offers a wide range of trial solutions to a problem, and natural selection, which picks the best and discards the others. Iterated repeatedly, these processes combine to create artefacts of extraordinary delicacy and ingenuity, from the human eye to the gecko's foot.

The traditional engineering approach is very different, designing not by scattershot experimentation and careful sifting but by rational planning. That, on the whole, is a better option - imagine trying to make an automobile by random assembly of randomly machined components. Darwinian evolution, lacking the luxury of foresight, achieves its wonders only because it has large populations and geological timescales to work on. But rational design isn't always possible: we might not understand the properties of the components or the target well enough, or the combinatorial universe of potential choices may be too vast. Both are sometimes true in materials science, for example in the case of compound superconductors. That's why, in this and other cases, something akin to Darwin's approach has been tried.

Combinatorial materials synthesis may have an evolutionary aspect when iterated: some selection criterion is used to extract

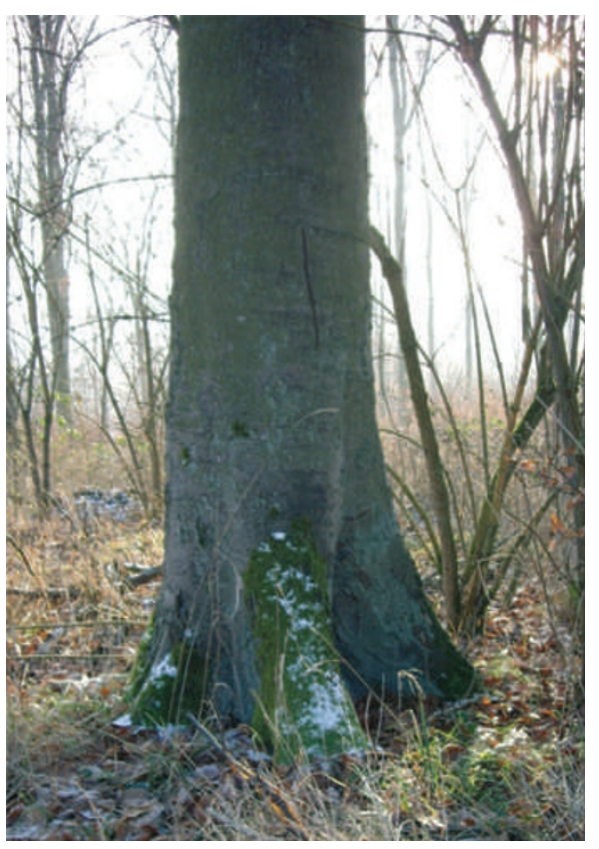

Trees avoid sharp-cornered notches by growing wood in a shape that reduces concentrations of tensile stress.

good candidates that are then reshuffled in the next round. That works well for in vitro methods of making biomolecules with new functions, but is rarely attempted in materials. It is, however, more or less the basis of Monte Carlo simulatedannealing methods in computer simulation, where some 'cost function' stands in for 'fitness' to evaluate the trial-anderror reorganization of the constituents. Genetic algorithms for surveying energy landscapes are particularly useful for the recalcitrant problem of first-principles structure prediction ${ }^{1}$.

Some examples of materials design stake a stronger claim to Darwinian methodology. Claus Mattheck of the Karlsruhe Institute of Technology has long championed the tree as a selfadjusting materials system that 'finds' a good structural response to stress ${ }^{2}$. The 'evolution' of this system is guided by cell responses to stress, rather than randomly varied, but the resulting shapes can serve as inspiration for a more strictly evolutionary route enacted by computation. Mattheck and co-workers have used this idea to make notch structures with little propensity to initiate cracks. In a related approach, Paolo Vannucci, Angela Vincenti and their colleagues in France have devised a genetic algorithm called BIANCA for optimizing a given property of a laminate: for example, zero or isotropic thermal expansion coefficients, maximum buckling stiffness, or a particular piezoelectric deformation ${ }^{3-5}$.

And Jim Platts of Cambridge University has used iterative computational evolution to deduce the optimal placement of fibres in a composite material ${ }^{6,7}$. Whereas Mattheck takes inspiration from wood, Platts and colleagues look to bone, in which material is actively removed as well as added in the adaptive sculpting process. That the growth and morphology of bone can be seen as a design process involving minimization was first recognized by the Australian engineer A. G. M. Michell in 1904, but his perceptive insight languished until sufficient computer power became available to make use of it.

Minimization problems like this are very hard from any analytical first-principles perspective. An evolutionary geneticalgorithm search of the design space is not truly Darwinian, because the target property is pre-determined rather than emerging as a consequence of selection by efficiency of replication. In that sense, it is a form of directed evolution, more akin to the selective breeding by the livestock managers and pigeon fanciers that proved so informative to Darwin. But it shares with true Darwinism the use of a population spread all over the search space, and the intelligent exploitation of information about that space by recombining aspects of both 'bad' and 'good' designs to guide the search towards an optimal solution. The great man would doubtless approve.

\footnotetext{
References

1. Woodley, S. M. \& Catlow, R. Nature Mater. 7, 937-946 (2008).

2. Mattheck, C. Secret Design Rules of Nature (Verlag Forschungszentrum Karlsruhe, 2007).

3. Vincenti, A. et al. Proc. 14th Int. Conf. Compos. Mater. Paper 1341 (2003).

4. Vannucci, P. Int. J. Struct. Mulidisc. Optimis. 31, 378-387 (2006).

5. Vannucci, P. \& Vincenti, A. Compos. Struct. 79, 454-466 (2007).

6. Platts, M. J. in Nature and Design (eds Collins, M. W. et al.) 157-167 (WIT Press, Southampton, 2005).

7. Platts, M. J. Paper presented at Opticon ' 99 Optimisation Software Methods \&Applications Conf., Newport Beach, October 1999.
} 